\title{
A Hybrid Method for Assigning Containers to AGVs in the Dynamic Environment of Container Terminals
}

\author{
Radhia ZAGHDOUD ${ }^{1,2}$, Khaled MESGHOUNI ${ }^{2}$, Simon Collart DUTILLEUL ${ }^{3}$, \\ Kamel ZIDI $^{1}$, Khaled GHEDIRA ${ }^{1}$ \\ ${ }^{1}$ SOIE: Laboratoire de Stratégies d'Optimisation et Informatique intelligente \\ Institut Supérieur de Gestion de Tunis, \\ 41, Rue de la Liberté, Cité Bouchoucha 2000 Le Bardo, Tunis -TUNISIE \\ Radhia.Zaghdoud@fsg.rnu.tn, k_zidi@ut.edu.sa, khaled.ghedira@isg.rnu.tn \\ ${ }^{2}$ LAGIS: Laboratoire d'Automatique, Génie Informatique et Signal \\ Ecole Centrale de Lillle, \\ Cité scientifique - B.P. 48-59651 Villeneuve d'Ascq, Lille-France \\ khaled.mesghouni@ec-lille.fr \\ ${ }^{3}$ ESTAS: Laboratoire d'Évaluation des Systèmes de Transports Automatisés et de leur Sécurité, de \\ l'aménagement et des réseaux \\ IFSTTAR Institut français des sciences et technologies des transports, \\ 20 rue Elisée RECLUS BP 70317, F-59666 Villeneuve d'Ascq Cedex, Lille-France \\ simon.collart-dutilleul@ifsttar.fr
}

\begin{abstract}
The handling operations performed in ports require the use of equipment operating in a dynamic environment. Some tasks may not be fully carried out due to equipment failure or power breakdown that may occur particularly with the automated guided vehicles (AGV). The unavailability of equipment such as AGV has important consequences in terms of respecting the deadlines of different operations that a port should perform, such as the loading and unloading operations of ships. This situation can aggravate if there are also traffic problems in the port with some inaccessible network nodes. A part of the equipment will be blocked or the operations will take longer than expected if they don't take the optimal path to connect the loading/unloading points and storage areas. These reasons confirm the usefulness of establishing a robust system able to resolve the problem of assigning containers in the static and dynamic environments. In a previous work, we developed a system for assigning containers in a static environment. In order to improve this method, we devote this paper to the study of the robustness of our system to the dynamic environment of the port. The numerical tests included in this paper show an adequate performance of our method for this particular dynamic environment.
\end{abstract}

Keywords: Assignment, Dynamic, AGV, Containers, Optimization, Genetic Algorithm.

\section{Introduction}

The maritime transportation has a great importance in global manufacturing and international business not only because it's the cheapest transportation way, but also because it has a capacity to transport a huge volume of goods. Since the 1960s, with the appearance of containerization, this field has received an important development. It increases the goods transportation speed and the goods volume. As a result of this development, a new container terminal model was erase built and the existing ones were extended. In the Hong Kong terminal container, turnover has been raised from 9 million twenty feet Equivalent Units (TEU) in 1993 to 19 million TEU in 2002[1]. The number of TEUs in Singapore, as the second port around the world, has been doubled, increasing from 9 million to 18 million TEUs [2]. But the seaports sizes increase has a negative effect on ship loading and unloading operations rapidity because it makes it very slow. As a consequence, the ship has to wait for a longer time at the port. Facing the challenge of the big increase of the container's number, the transportation systems of container terminals have to minimize the loading and unloading operation duration. In order to perform this operation, the researchers in this field have to decompose it in some subproblems such as storage containers problem, scheduling AGVs and quay cranes problems. Due to the big number of equipements, the environment in the container terminal is uncertain and complexe. All the operations are integrated, so each operation depends on several constraints. The AGVs scheduling problem is considered as multi-objective, uncertain and complexe. It is proved to be NP-hard problem. Given its large scale, the solution of this problem is not an optimal but near optimal solution. To solve this problem, the heuristic algorithms are widely used. Because of container terminal operation complexity, it's difficult to optimize the whole operation system with a single analytical model. Therefore, generally the operation system in 
container terminal is divided into several subprocesses and each sub-process is optimized separetly. This paper is organized as follows. The second section studies the literature review of the AGVs and containers scheduling in a dynamic enviroment. In the third section we propose the problem description. The fourth section describe the robustness of the proposed approach in the dynamic environment. Finally the conclusion and perspectives will be presented in the fifth section.

\section{Literature Review}

According to [3], the AGV scheduling approaches can be divided into two classes: offline scheduling and on-line scheduling. The off-line scheduling refers to the planning of activities for the entire scheduling period in advance. On the other hand, the on-line scheduling attempts to schedule operations dynamically at runtime. The solution time efficiency of an off-line approach is not a critical concern. This approach formulates machines and AGV scheduling problems as a constrained optimization model, and then solves the problem using an appropriate optimization algorithm. In the off-line approach, transportation requests, operation times and additional information about the system need to be perfectly predictable in advance. The off-line scheduling has attracted many researchers to find a separate schedule for operations and material handling systems or simultaneous schedule. Heuristic optimization approaches, are also used for a feasible and good solution of off-line scheduling problems as most problems are NP hard in terms of complexity [4]. In practice, exact information about jobs is usually known at a very late instant. This makes off-line scheduling hardly possible. Therefore, on-line scheduling or dynamic dispatching rules are necessary to control vehicles and machines $[5,6]$ one type of scheduling strategy is to use dynamic dispatching rules to determine which job should be processed next, when a resource becomes available. Such rules are very common in systems where many scheduling decisions must be made in a period of time. They consider an on-line dispatching system when a vehicle drops off a load or a new load arrives. There are two main types of on-line dispatching system; decentralized and centralized systems. If a single control system simultaneously controls all AGVs in the system, they refer it as a centralized system. All information related to vehicles such as pick-up and drop-off locations, load release times, vehicle positions and status are stored in the controller's data base [7]. For [8], if the dispatching decision is carried out, a route and schedule should be planned for the AGV to move the job from its origin to its destination within the AGV network. A route implies the path which should be taken by the AGV when making a pick-up or delivery. The related schedule gives arrival and departure times of the AGV at each part, pick-up and drop-off point and intersection during the route to ensure collision free routing. The selection of a certain route and schedule is effective in the performance of the system. Therefore, one of the objectives of the routing of AGVs is to minimize transportation times. The algorithms have to be developed to solve the routing problem. Two categories of algorithms can be distinguished, namely static and dynamic algorithms. Analogies between these problems from transportation literature and routing and scheduling problems for AGVs in automated guided vehicle systems are clear. Therefore, attention is paid in the literature to developing non-conflicting routes for AGVs. With a nonconflicting route, an AGV arrives as early as possible at the destination without conflicting with other AGVs. In AGV routing with static algorithms the route from node $i$ to node $j$ is determined in advance and is always used if a load has to be transported from $\mathrm{i}$ to $\mathrm{j}$. In this way, a simple assumption is to choose the route with the shortest distance from $\mathrm{i}$ to $\mathrm{j}$. However, these static algorithms are not able to adapt to changes in the system and traffic conditions. In dynamic routing, the routing decision is made based on real-time information and, as a result, various routes between $i$ and $j$ can be chosen. Static routing problems in $\mathrm{AGV}$ systems are related to vehicle routing problems (VRP) studied in transportation literature.

\section{Problem Description}

Assume a set of containers stored in different positions named depots at the port. These containers must be transported to a discharging location in order to be transferred by trucks, trains...etc to clients or to a charging location in order to be transferred to the ship. The problem consists to transporting these containers from an initial position to a final position by a set of AGVs. But the number of 
AGVs is limited and it's very small in comparison with containers number. So each AGV must transport a set of containers not once but at multiple travels. It's a repetitive task for each $\mathrm{AGV}$, this tasks consists in moving to the initial container position and transporting it to its final position. This work has to be the same for all the AGVs. The static version of this problem was studied in our previous work [9] and the numerical results showed the performance of the proposed approach. But in the real environment, where the conditions are changing, the model of port is bigger and the movement in the terminal is dynamic, the precedent approach will be unable to solve the problem.

\subsection{Main objective problem}

Our problem is included in a global problem; loading and unloading operation of a ship, in a container terminal. It is a problem of assigning and optimizing of the using of AGV. The main objective is to move all ship containers from a drop-off location to a pick-up location or backwards, with certain constraints; a minimum total time of work for all the system AGVs, a minimum time taken by the whole operation and a maximum equilibrium in time of work between AGVs. The assigning of a set of tasks to each AGVs depends on its availability. A task is defined as; transportation of only one container from a discharging location to a charging location or backwards. Throughout this period of time the AGV is not available, but after completing a task, an AGV can start another task. Throughout the whole operation of transporting, the $\mathrm{AGV}$ can be currently: (1) Available and in the same position of the container, so it can transport it directly. (2) Available but far from the position of the container, in this case, it must move to the position of the container in order to transport it. In order to optimize this operation, we propose to assign each task to the nearest available $\mathrm{AGV}$ and the $\mathrm{AGV}$ having the minimum total time of work. It must take the shortest path to arrive at the container position and move it to its final position. The AGV is autonomous but its autonomy is limited by the quantity of energy that it has. So it's necessary to optimize its work. That's why; we propose to create equilibrium between the working times of AGVs. The optimization of assigning containers to AGV depends on the distance between the AGV position and the initial container position, on its speed, on its tardiness caused by traffic routes and the difference between work times taken by each AGV of the system. When the AGV arrives at the container position it needs a special tool to dispose the container, it's generally called crane quay. For our application this crane quay is replaced by an instrument named cassette that's usually available to take the container on its position at the $\mathrm{AGV}$, this cassette can minimize the waiting time for container to become available. Containers on cassettes are thus disconnected from the transport equipment moving them, rendering higher efficiency and productivity since the idle time is reduced. So the AGV doesn't need to wait for the availability of a crane quay. The mathematic model was proposed in [9] and a multi-objective approach in a static environment was proposed in [10]. The objective of this work is to study the robustness of the approach proposed in $[9,10]$ to resolve the problem of assigning containers to AGVs in a big port with dynamic environment. In particular case, when some new containers arrive at the terminal and must be transported in urgent time. Also when some nodes are unavailable, the shortest paths may be unavailable too, so the necessity to replace this paths by others which are obligatory not optimal because the shortest paths are unique. So our exact objective in this work is to propose a robust system able to solve the problem of inserting new containers and exchanging the failed nodes or failed depots during the approach execution.

\subsection{Mathematic model}

$D=\left\{D_{1}, D_{2}, D_{3}, \ldots, D_{m}\right\} \quad$ a set of depots (loading and unloading depots) with a cardinality equal to $\mathrm{m}$.

$D^{\prime}=\left\{D_{1}^{\prime}, D^{\prime}{ }_{2}, D^{\prime}{ }_{3}, \ldots, D_{\mathrm{m}^{\prime}}\right\} \quad$ a set of new depots corresponding of new containers.

$T=\left\{T_{1}, T_{2}, T_{3}, \ldots, T_{n}\right\}$ a set of tasks with a cardinality equal to $n$.

$T^{\prime}=\left\{T_{1}^{\prime}, T_{2}^{\prime}, T^{\prime}, \ldots, T^{\prime}{ }^{\prime}\right\}$ a set of new tasks with a cardinality equal to $n$ '.

$V=\left\{V_{1}, V_{2}, V_{3}, \ldots, V_{k}\right\} \quad$ a set of vehicles (AGV) with a cardinality equal to $\mathrm{k}$.

$N I=\left\{N I_{1}, N I_{2}, N I_{3}, \ldots, N I_{p}\right\} \quad$ a set of intermediate nodes with a cardinality equal to $\mathrm{p}$.

$N I^{\prime}=\left\{N I_{1}, N I_{1}, N I_{1}, \ldots, N I^{\prime}{ }_{p}{ }^{\prime}\right\}$ a set of new intermediate nodes with a cardinality equal to $\mathrm{p}$ '. 
$N=N I \cup D$ a set of nodes representing depots nodes and intermediate nodes with a cardinality equal to $\mathrm{m}+\mathrm{p}$.

$N^{\prime}=N \cup N I^{\prime} \cup D^{\prime}$ a set of total number of nodes with new tasks and new nodes.

$d_{i j}$ : distance between node $\mathrm{i}$ and node $\mathrm{j}$.

$t_{i j v}$ : time taken by a vehicle $\mathrm{v}$ to go from node $\mathrm{i}$ to node $\mathrm{j}$.

$\left[I d_{i}, I f_{i}\right]$ : time windows of task.

$\left[I d_{i}+\tau_{i}, I f_{i}+\tau_{i}\right]:$ new time windows of task.

$d a_{i}$ : arrival time at node $\mathrm{i}$.

$d a_{i}+\tau{ }_{i}^{\prime}$ new arrival time at node $\mathrm{i}$.

$d d_{i}:$ departure time from node $\mathrm{i}$.

$d d_{i}+\tau^{\prime \prime}{ }_{i}$ : new departure time from node $i$.

$D a_{i}$ : lateness time at node $\mathrm{i}$.

$D a_{i}+\tau{ }^{\prime \prime}{ }_{i}$ : new lateness time at node $\mathrm{i}$.

$q_{i v}$ : load of vehicle $\mathrm{v}$ at node $\mathrm{i}$, equal to 1 or 0.

$D T_{v}$ : total time taken by vehicle $\mathrm{v}$ to transport all tasks.

dtv: total time taken vehicle $\mathrm{v}$ to transport the new tasks

$\mathrm{k}$ : total number of tasks

k1: total number of new tasks

$X_{i j v}$ : a decision variable

$\begin{cases}1 & \text { if vehicle } \mathrm{v} \text { is used } \\ 0 & \text { otherwise }\end{cases}$

The objective function of the problem is in equation (1)

This function is the aggregation of three functions which minimize respectively; the total time of work for all AGVs, the due time which AGV takes to leave an origin and to arrive at a destination and the equilibrium of working time between AGVs. The function value depends on $\alpha, \beta, \gamma$ values which can be fixed by a specialist in the seaport.
Such as:

$$
\begin{aligned}
& \sum_{i \in N} \sum_{v \in V} X_{i j v}=1, \quad \forall j \in N \\
& \sum_{i \in N} X_{i 0 v}=1, \quad \forall v \in V \\
& \sum_{j \in N} X_{0 j v}=1, \quad \forall v \in V \\
& \sum_{i \in N} X_{i j v}-\sum_{i \in N} X_{j i v}=0, \quad \forall v \in V, \forall j \in N \\
& Q_{v}=\sum_{i \in N} \sum_{j \in N} q_{i j v}=1, \forall v \in V \\
& X_{i j v}=1 \Rightarrow I d_{i}+\tau_{i} \leqslant d a_{i}+\tau^{\prime}{ }_{i} \leqslant I f_{i}+\tau_{i}, \\
& \quad \forall i, j \in N, \forall v \in V \\
& X_{i j v}=1 \Rightarrow I d_{i}+\tau_{i} \leqslant d d_{i}+\tau^{\prime \prime}{ }_{i} \leqslant I f_{i}+\tau_{i}, \\
& \quad \forall i, j \in N, \forall v \in V \\
& X_{i j v}=1 \Rightarrow d d_{i}+\tau^{\prime \prime}{ }_{i}+t_{i j v}+D a_{j} \leqslant I f_{j}, \\
& \quad \forall i, j \in N, \forall v \in V \\
& X_{i j v}=1 \Rightarrow d a_{j}-t_{i j v}+D a_{j} \leqslant I d_{j}, \\
& \forall i, j \in N, \forall v \in V
\end{aligned}
$$

(2) The transport cost from node $i$ to node $j$ is equal to 1

(3) and (4) explain the possibility to go from node zero to any other node

(5) each arc is bidirectional

(6) the charge of $\mathrm{AGV}$ is the equal to 1

(7) the AGV must arrive at node $i$ in the arriving time window of node

(8) the container must be moved in the departure time window of node

(9) and (10) AGV must arrive before beginning date time window of node and must move before the end of node time window.

\subsection{Proposed approach}

In this paper, we apply an hybridization between an Exact Algorithm Dijkstra, heuristic and Genetic Algorithm. The first algorithm is used to compute the shortest path travelled by each $\mathrm{AGV}$, the choice of $\mathrm{AGV}$ is resolved by

$$
\begin{aligned}
& F \equiv\left\{\begin{array}{l}
F 1=\sum_{i=1}^{i=N+N^{\prime}} \sum_{j=1}^{j=N+N^{\prime}} \sum_{v=1}^{v=V} X_{i j v} * d_{i j v} \\
F 2=\sum_{i \in N}\left(I d_{i}+\tau_{i}\right)-\left(d a_{i}+\tau_{i}^{\prime}\right)+\sum_{i \in N}\left(I f_{i}+\tau_{i}\right)-\left(d d_{i}+\tau^{\prime \prime}{ }_{i}\right) \\
F 3=\sqrt{\left(1 /\left(k+k_{1}\right)-1 /\left(k+k_{1}\right)^{3}\right) *\left(\sum_{v \in V}\left(D T_{v}+d t_{v}\right)^{2}-2 * \prod_{v \in V}\left(D T_{v}+d t_{v}\right)\right)}
\end{array}\right. \\
& F=\alpha * F_{1}+\beta * F_{2}+\gamma * F_{3}
\end{aligned}
$$


the heuristic and the Genetic Algorithm is used to optimize the dispatching of containers to AGVs, by its operators of cross-over and mutation. This approach is applied to calculate the global multi-objective function value which is the aggregation of three functions corresponding respectively to total working time, equilibrium of charge between AGVs and respect of task time window. The criterion of total working time is the time made by all AGVs to move all containers from their initial positions to their final positions. The equilibrium of $\mathrm{AGVs}$ charges criterion consists in minimizing the difference in working time between the AGVs, because the vehicle autonomous so it is very important to minimize its energy consumption. The respect of time windows criterion minimizes the delays of time on the operation of moving a container from its initial position to its final position.

\subsection{Hybrid approach: GA \& DIJK \& HEUR}

3. Choose a task by precedence constraint

4. Choose the nearest available AGV

5. Compute the shortest path for AGVs

6. Repeat steps 1 through 4 for the $\mathrm{N}$ tasks

7. Compute the objective function: $F_{\text {cost }}=\lambda * d_{\text {empty }}+\mu * d_{\text {full }}$

8. Generate random initial population by changing the initial positions of AGVs at the beginning of each solution

9. Apply the GA with its operator's selection, crossover and mutation

10. Correct whenever individuals are after crossover and mutation by computing the shortest path for dempty

11. Stop algorithm, when the solution becomes constant during an important number of generations

\subsection{Extended approach for tasks insertion}

The assignment of tasks to AGVs is by choosing the nearest available AGV through applying a heuristic and each AGV have to take the shortest path computed by Dijkstra algorithm. The initial population of the genetic algorithm is chosen randomly.

(step1 to step7): from GA \& DIJK \& HEUR

8. Insertion of new tasks
9. Update solutions and genetic algorithm population

\section{0. step 8 from GA \& DIJK \& HEUR}

\section{1. step 9 from GA \& DIJK \& HEUR}

\section{Experimental Studies}

When the movement in the port is very dynamic, especially if the number of ships arriving each day at the terminal is big, it is possible that some ships arrive with lateness. As a consequence, all other next ships will be unloaded in tardiness. The accumulation of time lateness can be big and it can have a serious consequence. An important problem caused by this tardiness, a ship arrives at the terminal and no equipment, no depot or storage zones are available for the unloading operation. This problem will be more serious if the containers have a transfer date priority. An urgent solution can be proposed to solve this problem is to create some temporary depots of some previous containers in order to accelerate the loading or unloading operations and have a storage zones available as soon as possible. To show the performance of our approach in this problem, in particular when the depots are not fixed in advance or fixed in advance but was changed during the operation of containers transportation, we make a comparison study of the approach convergence between two cases: when the depots are fixed in advance and when the depots positions are not fixed.

\subsection{Scenario}

The application of these approaches is made with a computer having $2 \mathrm{GO}$ of RAM memory and $1.5 \mathrm{GHz}$ processor speed. The language application is $\mathrm{C}++$. We tested the approaches with 20 tasks and 4 AGVs. The genetic algorithm parameters are: the probability of individuals selected from every population for crossing is 0.7 and the probability of individuals selected for mutation is 0.1 . For each case, we make the average of ten running tests and then we computed the objective function value variation with generation number variation. The matrix representing the distances between nodes in the port will be showed in Appendix.

\subsection{Non fixed depots}

The numerical tests in this case are made with the big port model described previously, 
because it is more possible to have the dynamicity in big port than in small one. The comparison study is made between two cases: In the first case, the depots are fixed in advance, but in the second case we choose randomly the depots of loading and unloading. This event can also happen when a vehicle is breakdown and is obliged to dispose the container in any position of the port. The depots fixed in advance must be changed and a new task appears which consist to transport the container from its current position to the final depot. The numerical results in Figure 1 show the convergence of the proposed approach with fixed depots and non fixed depots. These tests show the robustness of our approach with changeable depots positions.

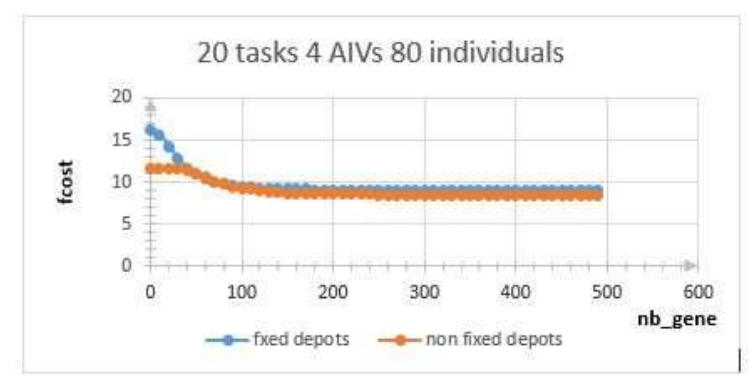

Figure 1: Results of GA \& DIJK \& HEUR Approach in big port

The application of our approach with fixed depots and not fixed depots shows an important difference in initial solution values. When the number of depots is fixed at 4 for example, the initial solution is equal to 16.2 , but for nonfixed depots, the initial solution value is equal to 12. Although this difference, our approach converges in the two cases after some generations. The convergence in the case of fixed depots is earlier than of non-fixed depots. But the final solution with non-fixed depots is better than the fixed depots solution.

\subsection{Non static number of tasks}

In the big port, any lateness of the loading or unloading operation of a ship will be translated to the next ships. This event may cause the arriving of some ships in the same time which can cause the non-existing of vehicles necessary for transporting containers. If the containers corresponding of a ship are urgent, we need to apply the procedure of containers insertion to vehicles in order to serve these containers as soon as possible.

These numerical results show the performance of our approach to adding a number of tasks during the genetic algorithm evolution. It shows the ability of this approach to converge to the best solution, after receding from the solution obtained before the insertion of tasks which is near the best solution. In comparison with the result of 30 tasks, the insertion of 10 tasks to the 20 initial tasks doesn't prevent it to converge. There is a difference in the best solution between the two cases: when the number of tasks is 30 from the beginning of the approach execution and when some new tasks are added during the approach execution.

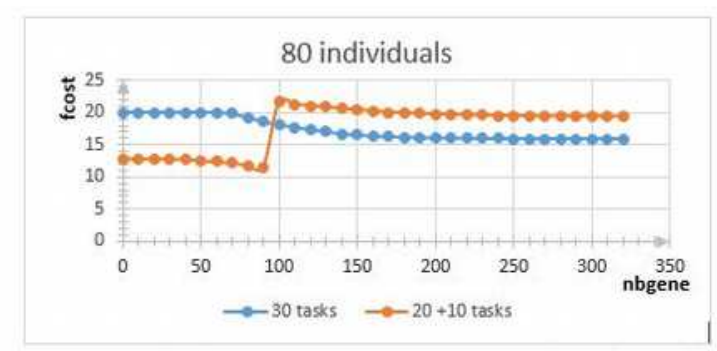

Figure 2: Results of GA \& DIJK \& HEUR Approach after tasks insertion

\subsection{Disturbance in the port network}

In the dynamic environment, during the approach execution, it is possible that some nodes failed, so the paths including these nodes are unavailable. In order to continue the approach execution, we propose to choose other nodes and other paths which are not obligatory the shortest. The genetic algorithm continues its generation after this event, in order to find the best solution by its operators of cross-over and mutation. We propose to use some random paths to replace the shortest paths unavailable. For example in this test, in the 50th generation of genetic algorithm, some nodes become unavailable; we exchanged it by others and continue the approach execution.

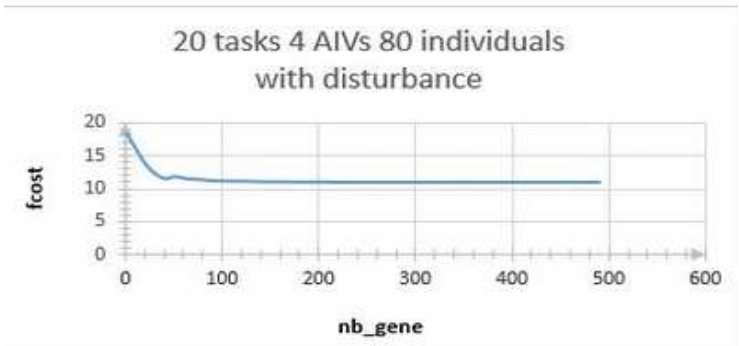

Figure 3. Results of GA \& DIJK \& HEUR

Approach before and after nodes exchanges

When there are some nodes unavailable following a disturbance, the shortest paths containing these nodes will be also unavailable. As a consequence, all the AGVs taking this path will be obliged to choose other path in order to move to the container initial position or to transport it to its final position. The solution obtained is not better than the solution without 
disturbance because in this case obviously, the shortest path is used. The initial solution with disturbance is better than the solution without disturbance, but rapidly, the solution without traffic network will be better. This amelioration is made because the operators of genetic algorithm through the generations. These numerical results show the performance of our approach to find the best solution also in the presence of network disturbance.

\section{Conclusion and Perspectives}

We propose in this work a hybrid approach: GA \& DIJK \& HEUR to solve the problem of assigning containers to AGVs in the dynamic enviromenet of a containers terminal. The real live in the port is not usually static; it's very dynamic specially when there is a big port. In order to propose an efficient system, we studied the robustness of our system in a dynamic environment. The dynamic environment of port can be in different forms: the non fixed depots and the non fixed number of containers transported by each AGV and the change of network port state. For each case, our proposed approach was extended to be adaptable to the environment modification. The numerical results show a good performance of our proposed approach for all cases. By analogy to this transport system in a terminal containres, we propose the idea to apply this approach to transport containers in the airport. There are some AGVs which transport the baggages or containers from the flight position to the airport or inversely. Each vehicle has a path to travel, this path must be optimal in order to minimize the transport time. Also each vehicle has a set of bags to transport, but in general all these bags must be taken from the one position to the one destination because a vehicle can transport only the bags corresponding to one flight. The important difference with maritime port is the high degree of dynamicity in the airport.

\section{REFERENCES}

1. STEENKEN, D., S. VOB, R. STAHLBOCK, Container Terminal Operation and Operations Research - A Classification and Literature Review, Operational Research Spectrum, vol. 26, 2004, pp. 3-49.

2. RASHIDI, I. H., E. P. K. TSANK, Container Terminals: Scheduling Decisions, Their Formulation and
Solutions, submitted in Journal Scheduling and Engineering, 2006.

3. EROL, R., C. SAHIN, A. BAYKASOGLU, V. KAPLANOGLU, A Multi-agent based Approach to Dynamic Scheduling of Machines and Automated Guided Vehicles in Manufacturing Systems. Applied Soft Computing, vol. 12(6), 2012, pp. 1720-1732.

4. FISHER, M., Vehicle Routing, in Ball, M. O., Magnanti, C. L., Monma, C. L., Nemhauser, G. L. (eds.) Network Routing, Amsterdam, Elsevier, 1995, pp. 1-33.

5. LUCAS, B. B., R. R. EDILSON, E. KATO, A Multi-Agent System using Fuzzy Logic to Increase AGV Fleet Performance in Warehouses. International Conference on Fuzzy Systems, 2008, pp. 2291-2298.

6. GOTTING, H. H., Automation and Steering of Vehicles in Ports. Port Technology International, vol. 10, 2000, pp. 101-111.

7. FAZLOLLAHTABAR, H., N. MAHDAVIAMIRI, An Optimal Path in a Bi-criteria AGV-based Flexible Job-shop Manufacturing System Having Uncertain Parameters. International Journal of Industrial System Engineering, vol. 13(1), 2013, pp. 27-55.

8. MEERSMANS, P. J. M., A. P. M. WAGELMANS, Effective Algorithms for Integrated Scheduling of Handling Equipment at Automated Container Terminals. ERIM Report Series Research in Management ERS-2001-36-LIS, Erasmus University Rotterdam, 2001.

9. ZAGHDOUD, R., K. MESGHOUNI, S. C. DUTILLEUL, K. ZIDI, K. GHEDIRA, A Multi-objective Approach for Assignment Containers to AGVs in a Container Terminal, The 2013 IEEE International Conference, Manchester, UK 13 - 16 October 2013.

10. ZAGHDOUD, R., K. MESGHOUNI, S. C. DUTILLEUL, K. ZIDI, K. GHEDIRA, Optimization Problem of Assignment Containers to AGVs in a Container Terminal, 13th Symposium in Control in Transportation Systems, Bulgaria, 2012. 
Appendix - The matrix of distances computed with Dijkstra with 26 nodes

\begin{tabular}{|c|c|c|c|c|c|c|c|c|c|c|c|c|c|c|c|c|c|c|c|c|c|c|c|c|c|c|c|}
\hline & DD1 & DD2 & DD3 & DC1 & DC2 & DC3 & N1 & $\mathrm{N} 2$ & N3 & $\mathrm{N} 4$ & N5 & N6 & N7 & N7 & N8 & N9 & N10 & N11 & N12 & N13 & N14 & N15 & N16 & N17 & N18 & N19 & $\mathrm{N} 20$ \\
\hline DD1 & 0.0 & 12.0 & 20.0 & 36.7 & 48.7 & 51.8 & 5.4 & 7.8 & 17.8 & 11.2 & 12.8 & 24.2 & 20.2 & 19.2 & 22.8 & 20.8 & 26.8 & 26.8 & 32.2 & 37.2 & 27.2 & 28.8 & 40.2 & 43.2 & 33.0 & 33.8 & 43.8 \\
\hline DD2 & 12.0 & 0.0 & 8.0 & 45.2 & 40.2 & 40.4 & 17.4 & 7.8 & 6.4 & 23.2 & 12.8 & 12.8 & 31.8 & 27.8 & 22.8 & 20.8 & 24.1 & 24.1 & 20.8 & 25.8 & 35.8 & 28.8 & 28.8 & 31.8 & 41.6 & 33.8 & 35.2 \\
\hline DD3 & 20.0 & 8.0 & 0.0 & 50.7 & 40.2 & 35.5 & 25.4 & 15.8 & 6.4 & 31.2 & 20.8 & 12.8 & 39.8 & 35.8 & 30.8 & 28.8 & 24.1 & 24.1 & 20.8 & 18.7 & 43.8 & 34.1 & 28.8 & 26.9 & 47.1 & 39.1 & 35.2 \\
\hline DC1 & 36.7 & 45.2 & 50.7 & 0.0 & 12.0 & 20.0 & 31.3 & 37.4 & 44.3 & 25.4 & 32.4 & 37.9 & 18.4 & 17.4 & 22.4 & 24.6 & 26.6 & 26.6 & 31.4 & 34.6 & 9.4 & 16.6 & 23.4 & 26.4 & 3.6 & 11.6 & 17.0 \\
\hline $\mathrm{DC} 2$ & 48.7 & 40.2 & 40.2 & 12.0 & 0.0 & 8.0 & 43.3 & 41.0 & 33.8 & 37.4 & 36.0 & 27.4 & 30.4 & 29.4 & 34.0 & 28.0 & 27.4 & 27.4 & 19.4 & 22.6 & 21.4 & 20.0 & 11.4 & 14.4 & 15.6 & 15.0 & 5.0 \\
\hline DC3 & 51.8 & 40.4 & 35.5 & 20.0 & 8.0 & 0.0 & 51.0 & 43.9 & 33.9 & 45.4 & 41.5 & 27.5 & 38.4 & 37.4 & 39.5 & 33.5 & 27.5 & 27.5 & 19.5 & 16.8 & 29.4 & 25.5 & 11.5 & 8.5 & 23.6 & 23.0 & 13.0 \\
\hline N1 & 5.4 & 17.4 & 25.4 & 31.3 & 43.3 & 51.0 & 0.0 & 13.2 & 23.2 & 5.8 & 9.4 & 29.6 & 14.8 & 13.8 & 18.8 & 17.4 & 23.4 & 23.4 & 31.4 & 36.4 & 21.8 & 25.4 & 39.4 & 42.4 & 27.7 & 30.4 & 40.4 \\
\hline $\mathrm{N} 2$ & 7.8 & 7.8 & 15.8 & 37.4 & 41.0 & 43.9 & 13.2 & 0.0 & 10.0 & 19.0 & 5.0 & 16.4 & 24.0 & 20.0 & 15.0 & 13.0 & 19.0 & 19.0 & 24.4 & 29.4 & 28.0 & 21.0 & 32.4 & 35.4 & 33.8 & 26.0 & 36.0 \\
\hline N3 & 17.8 & 6.4 & 6.4 & 44.3 & 33.8 & 33.9 & 23.2 & 10.0 & 0.0 & 29.0 & 15.0 & 6.4 & 34.0 & 30.0 & 25.0 & 23.0 & 17.7 & 17.7 & 14.4 & 19.4 & 38.0 & 27.7 & 22.4 & 25.4 & 40.7 & 32.7 & 28.8 \\
\hline $\mathrm{N} 4$ & 11.2 & 23.2 & 31.2 & 25.4 & 37.4 & 45.4 & 5.8 & 19.0 & 29.0 & 0.0 & 15.3 & 35.4 & 8.9 & 8.0 & 13.0 & 19.0 & 25.0 & 25.0 & 33.0 & 38.0 & 16.0 & 21.6 & 35.6 & 38.6 & 21.8 & 26.6 & 36.6 \\
\hline N5 & 12.8 & 12.8 & 20.8 & 32.4 & 36.0 & 41.5 & 9.4 & 5.0 & 15.0 & 15.3 & 0.0 & 21.4 & 19.0 & 15.0 & 10.0 & 8.0 & 14.0 & 14.0 & 22.0 & 27.0 & 23.0 & 16.0 & 30.0 & 33.0 & 28.8 & 21.0 & 31.0 \\
\hline N6 & 24.2 & 12.8 & 12.8 & 37.9 & 27.4 & 27.5 & 29.6 & 16.4 & 6.4 & 35.4 & 21.4 & 0.0 & 32.3 & 28.3 & 23.3 & 17.3 & 11.3 & 11.3 & 8.0 & 13.0 & 36.3 & 21.3 & 16.0 & 19.0 & 34.3 & 26.3 & 22.4 \\
\hline N7 & 20.2 & 31.8 & 39.8 & 18.4 & 30.4 & 38.4 & 14.8 & 24.0 & 34.0 & 8.9 & 19.0 & 32.3 & 0.0 & 4.0 & 9.0 & 15.0 & 21.0 & 21.0 & 29.0 & 34.0 & 8.9 & 17.6 & 31.6 & 34.6 & 14.8 & 22.6 & 32.6 \\
\hline N8 & 19.2 & 27.8 & 35.8 & 17.4 & 29.4 & 37.4 & 13.8 & 20.0 & 30.0 & 8.0 & 15.0 & 28.3 & 4.0 & 0.0 & 5.0 & 11.0 & 17.0 & 17.0 & 25.0 & 30.0 & 8.0 & 13.6 & 27.6 & 30.6 & 13.8 & 18.6 & 28.6 \\
\hline N9 & 22.8 & 22.8 & 30.8 & 22.4 & 34.0 & 39.5 & 18.8 & 15.0 & 25.0 & 13.0 & 10.0 & 23.3 & 9.0 & 5.0 & 0.0 & 6.0 & 12.0 & 12.0 & 20.0 & 25.0 & 13.0 & 14.0 & 28.0 & 31.0 & 18.8 & 19.0 & 29.0 \\
\hline N10 & 20.8 & 20.8 & 28.8 & 24.6 & 28.0 & 33.5 & 17.4 & 13.0 & 23.0 & 19.0 & 8.0 & 17.3 & 15.0 & 11.0 & 6.0 & 0.0 & 6.0 & 6.0 & 14.0 & 19.0 & 19.0 & 8.0 & 22.0 & 25.0 & 21.0 & 13.0 & 23.0 \\
\hline N11 & 26.8 & 24.1 & 24.1 & 26.6 & 27.4 & 27.5 & 23.4 & 19.0 & 17.7 & 25.0 & 14.0 & 11.3 & 21.0 & 17.0 & 12.0 & 6.0 & 0.0 & 0.0 & 8.0 & 13.0 & 25.0 & 10.0 & 16.0 & 19.0 & 23.0 & 15.0 & 22.4 \\
\hline $\mathrm{N} 12$ & 32.2 & 20.8 & 20.8 & 31.4 & 19.4 & 19.5 & 31.4 & 24.4 & 14.4 & 33.0 & 22.0 & 8.0 & 29.0 & 25.0 & 20.0 & 14.0 & 8.0 & 8.0 & 0.0 & 5.0 & 33.0 & 18.0 & 8.0 & 11.0 & 31.0 & 23.0 & 14.4 \\
\hline $\mathrm{N} 13$ & 37.2 & 25.8 & 18.7 & 34.6 & 22.6 & 16.8 & 36.4 & 29.4 & 19.4 & 38.0 & 27.0 & 13.0 & 34.0 & 30.0 & 25.0 & 19.0 & 13.0 & 13.0 & 5.0 & 0.0 & 38.0 & 23.0 & 11.2 & 8.2 & 35.6 & 27.6 & 17.6 \\
\hline N14 & 27.2 & 35.8 & 43.8 & 9.4 & 21.4 & 29.4 & 21.8 & 28.0 & 38.0 & 16.0 & 23.0 & 36.3 & 8.9 & 8.0 & 13.0 & 19.0 & 25.0 & 25.0 & 33.0 & 38.0 & 0.0 & 18.8 & 30.2 & 33.2 & 5.8 & 13.8 & 23.8 \\
\hline N15 & 28.8 & 28.8 & 34.1 & 16.6 & 20.0 & 25.5 & 25.4 & 21.0 & 27.7 & 21.6 & 16.0 & 21.3 & 17.6 & 13.6 & 14.0 & 8.0 & 10.0 & 10.0 & 18.0 & 23.0 & 18.8 & 0.0 & 14.0 & 17.0 & 13.0 & 5.0 & 15.0 \\
\hline N16 & 40.2 & 28.8 & 28.8 & 23.4 & 11.4 & 11.5 & 39.4 & 32.4 & 22.4 & 35.6 & 30.0 & 16.0 & 31.6 & 27.6 & 28.0 & 22.0 & 16.0 & 16.0 & 8.0 & 11.2 & 30.2 & 14.0 & 0.0 & 3.0 & 24.4 & 16.4 & 6.4 \\
\hline N17 & 43.2 & 31.8 & 26.9 & 26.4 & 14.4 & 8.5 & 42.4 & 35.4 & 25.4 & 38.6 & 33.0 & 19.0 & 34.6 & 30.6 & 31.0 & 25.0 & 19.0 & 19.0 & 11.0 & 8.2 & 33.2 & 17.0 & 3.0 & 0.0 & 27.4 & 19.4 & 9.4 \\
\hline $\mathrm{N} 18$ & 33.0 & 41.6 & 47.1 & 3.6 & 15.6 & 23.6 & 27.7 & 33.8 & 40.7 & 21.8 & 28.8 & 34.3 & 14.8 & 13.8 & 18.8 & 21.0 & 23.0 & 23.0 & 31.0 & 35.6 & 5.8 & 13.0 & 24.4 & 27.4 & 0.0 & 8.0 & 18.0 \\
\hline N19 & 33.8 & 33.8 & 39.1 & 11.6 & 15.0 & 23.0 & 30.4 & 26.0 & 32.7 & 26.6 & 21.0 & 26.3 & 22.6 & 18.6 & 19.0 & 13.0 & 15.0 & 15.0 & 23.0 & 27.6 & 13.8 & 5.0 & 16.4 & 19.4 & 8.0 & 0.0 & 10.0 \\
\hline $\mathrm{N} 20$ & 43.8 & 35.2 & 35.2 & 17.0 & 5.0 & 13.0 & 40.4 & 36.0 & 28.8 & 36.6 & 31.0 & 22.4 & 32.6 & 28.6 & 29.0 & 23.0 & 22.4 & 22.4 & 14.4 & 17.6 & 23.8 & 15.0 & 6.4 & 9.4 & 18.0 & 10.0 & 0.0 \\
\hline
\end{tabular}

